\title{
REGULARIZATION OF FEEDWATER FLOW RATE EVALUATION FOR VENTURI METER FOULING PROBLEM IN NUCLEAR POWER PLANTS.
}

\author{
Andrei V. Gribok, Ibrahim Attieh, J. Wesley Hines, Robert E. Uhrig \\ Nuclear Engineering Department \\ The University of Tennessee \\ Knoxville, TN 37996
}

agribok@utk.edu iattieh@utkux.utcc.utk.edu hines@utkux.utcc.utk.edu ruhrig@utk.edu

\section{KEY WORDS}

Regularization, inferential sensing, venturi meter, fouling, least squares solution

\begin{abstract}
Inferential sensing is a method which can be used to evaluate parameters of a physical system based on a set of measurements related to these parameters. The most common method of inferential sensing uses mathematical models to infer a parameter value from correlated sensor values. However, since inferential sensing is an inverse problem, it can produce inconsistent results due to minor perturbations in the data. This research shows that regularization can be used in inferential sensing to produce consistent results. The important example of monitoring nuclear power plant feedwater flow rate is given using data from Florida Power Corporation's Crystal River Nuclear Power Plant.
\end{abstract}

\section{INTRODUCTION}

Inferential sensing is the prediction of a plant variable through the use of correlated plant variables. A correct prediction of a variable can be used to monitor sensors which measure that variable, for drifts or failures making periodic instrument calibrations unnecessary. This move from periodic to continuous monitoring permits condition based maintenance that can reduce costs and increase the reliability of the instrument. Having accurate, reliable measurements is important for signals that may impact safety or profitability. One of these signals in nuclear power plants (NPP) is feedwater flow rate which is directly involved in the estimation of the thermal power of a reactor. Both PWRs and BWRs use venturi meters to measure feedwater flow rate to the steam generator and the pressure vessel, respectively. These meters are sensitive to measurement degradation due to corrosion products in the feedwater. Measurement error due to feedwater fouling, results in feedwater flow rate overestimation. Consequently, the thermal power of the reactor is also overestimated, and the reactor must be derated to stay within regulatory limits.

To overcome this problem, an inferential sensing system is being developed at the University of Tennessee to infer the "true" feedwater flow rate. This system infers values of 
complex process variables by integrating information from multiple sensors. A least squares model (linear or nonlinear) can be "trained" to map appropriate input variables to the desired output. Because inferential sensing is an inverse problem, it is often an ill-posed problem. Illposed problems suffer from lack of uniqueness and/or stability of the solution due to small perturbations in the data. This fact raises concerns about the consistency of inferential measurements. This paper shows that regularization techniques can be effectively used to provide a stable solution for inferential sensing, thus providing a stable, consistent estimation of feedwater flow rate. This estimation can then be used in the calculation of reactor thermal power to avoid unnecessary derating.

\section{FEEDWATER FLOW MEASUREMENTS IN U.S. NUCLEAR POWER PLANTS AND FOULING PROBLEM.}

In the United States, a nuclear power plant's operating limit is directly related to its thermal power production. The simplified energy balance equation can be written as (Chan and Ahluwalia, et al., 1992):

$$
\mathrm{Q}_{\mathrm{c}}=\mathrm{m}_{\mathrm{fw}} *\left(\mathrm{~h}_{\mathrm{s}}-\mathrm{h}_{\mathrm{fw}}\right)+\text { Losses, }
$$

where $\mathrm{Q}_{\mathrm{c}}$ is core thermal power, $\mathrm{h}_{\mathrm{s}}$ and $\mathrm{h}_{\mathrm{fw}}$ are enthalpies of steam and feedwater, respectively, and $m_{\mathrm{fw}}$ is feedwater flow rate.

Since the enthalpies of steam and feedwater can be determined accurately, uncertainties in thermal power estimation often come from feedwater flow rate measurements (Chan and Ahluwalia, et al., 1992). The majority of PWRs and some BWRs utilize venturi meters to measure feedwater flow rate because of their ruggedness and precision. However, these meters are susceptible to measurement drift due to corrosion products in the feedwater building up on the meter's orifice. This increases the measured pressure drop across the meters, which results in an over-estimation of the flow rate. Consequently, the reactors' thermal power is also overestimated (Chan and Ahluwalia, et al. 1992). A schematic drawing of a venturi meter and its fouling zone, or region of corrosion product buildup, is shown in Figure 1.

To stay within regulatory limits, reactor operators are forced to derate their plants. According to Chan and Ahluwalia, et al. (1992), venturi meter fouling is "the single most frequent cause" for derating in PWRs. The amount of derating, according to the report, varied from insignificant to $3 \%$ of full power. On average, the derating was between $1 \%$ and $2 \%$ of full power. A derating of $2 \%$ in an 800 MWe unit will cost the utility approximately $\$ 20,000$ per day given the cost of electricity is $\$ 0.05 / \mathrm{kWh}$. 


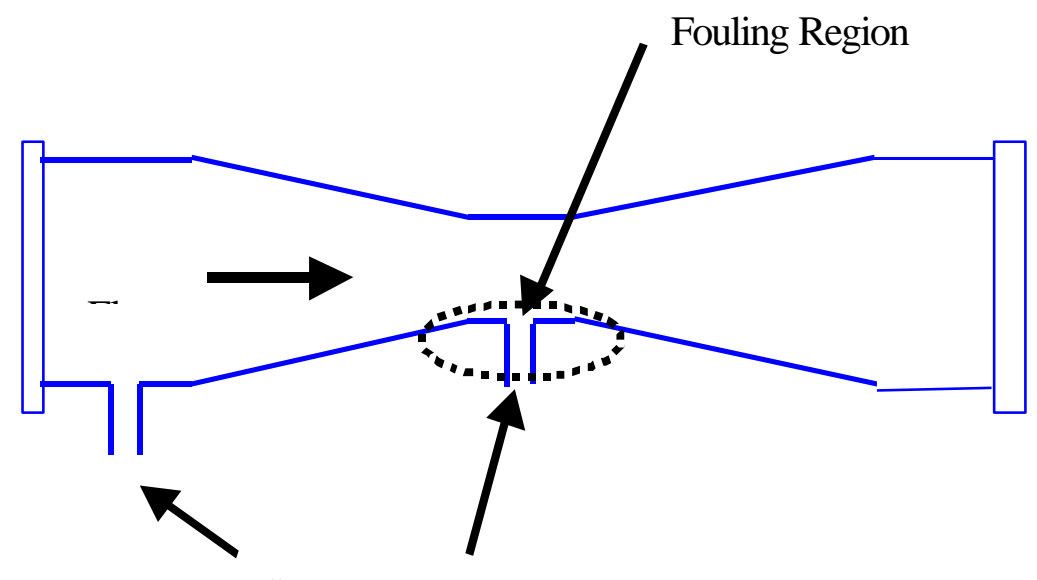

Sensor Ports

Fig.1 Schematic of a venturi meter

The most common practice at PWRs is to inspect and clean the venturi meters between fuel cycles. A major problem with this technique is that deposits can reappear as quickly as a month after achieving $100 \%$ reactor power (Chan and Ahluwalia, et al., 1992). To overcome this fouling problem, some utilities have developed a fouling coefficient or a correction factor to offset the degradation in the measurements' accuracy, and assume a linear increase for the first several months. Currently, ultrasonic flowmeters are considered to be a competitive alternative to venturi meters because they do not suffer from this fouling problem. However, they need further development to improve their accuracy and reliability.

The primary goal of this paper is to show how inferential measurements can be used to estimate feedwater flow rate, and how regularization can be used to make this estimation robust and consistent.

\subsection{Inferential Measurements for Feedwater flow estimations.}

Despite the susceptibility of the venturi meter to fouling, it is still the most common flow measurement instrument used in nuclear power plants. The University of Tennessee's Nuclear Engineering Department has been researching the development of inferential measurement or inferential sensing systems since the late eighties. These inferential systems utilize statistical or artificial intelligence based methods for predicting variables that are difficult or expensive to measure. One paper that dealt with venturi meter fouling was published by Kavaklioglu and Upadhyaya (1994). The development of an inferential sensing system consists of collecting training and testing data, preprocessing the data to remove outliers, and scaling the data to allow the use of statistical signal evaluation techniques. Once the data is collected and preprocessed, the inferential model is developed and tested. Several predictive modeling techniques can be applied to inferential sensing including linear techniques such as regression, principal component regression, ridge regression, and partial least squares; and non-linear techniques, such as nonlinear regression, non-linear partial least squares, and artificial neural networks. 
Inferential measurements should not be confused with classical prediction where a parameter value is estimated at time $t+1$, based on information about other parameters at time t. In inferential measurements, a parameter is estimated at time $t$ based on information about other parameters also at time t. Thus, the unknown value is "inferred" at the same time stamp. However, this does not make inference an easier problem than prediction. In fact, inferential measurement is often an ill-posed problem which requires special considerations.

\section{ILL-POSED PROBLEMS}

In 1923 the French mathematician Hadamard introduced the notions of well-posed and ill-posed problems, (Hadamard, 1923). Hadamard defined a well-posed problem as a problem which satisfies the following three conditions:

1. The solution for the problem exists (existence)

2. This solution is unique (uniqueness)

3. This solution is stable or smooth under small perturbations of the data, i.e. small perturbations in the data should produce small perturbations in the solution (stability).

If one of these conditions is not met, the problem is said to be ill-posed and requires special considerations. The existence of the solution can be enforced by enlarging the solution space. An example is the solution of polynomial equations. It is a well known fact from the basic theorem of algebra that any polynomial equation of order $\mathrm{n}$ has exactly $\mathrm{n}$ roots, real or complex. However, not all polynomial equations have solutions in the real domain, but they do have solutions in the complex domain. Thus, to guarantee the existence of the solution we have to enlarge the space within which we are seeking the solution.

Non-uniqueness usually arises when some of the information about the underlying model is lacking. Neural networks provide an excellent example of such non-uniqueness. Neural networks are essentially devices for building models from a finite amount of data. Due to the use of nonlinear transfer functions, neural network's error surfaces may contain many local minima. Assuming random weight and bias initialization, neural network training algorithms can provide a number of solutions (sets of weights), each of which fits the training data very well. Each possible solution will correspond to a different minima in the error surface, and thus, neural network's error surface presents a set of non-unique solutions. From this set of non-unique solutions, we must complete the difficult task of choosing a solution which adequately represents the modeled system.

Unstable solutions usually result when we are trying to reverse cause-effect relationships where the forward operator (the operator which solves the forward problem) is a smoothing operator. A smoothing operator attenuates high frequency oscillations in the input data. If the forward operator is a smoothing operator, then it is natural to expect that the inverse operator would be a "roughening" operator. A "roughening" operator amplifies high frequency oscillations, thus providing an unstable solution. An example of solution instability can be found in convolution-deconvolution relations. Convolution is a smoothing operator, hence deconvolution 
is a "roughening" operator. Deconvolution is a classic ill-posed problem which is very difficult to tackle without special methods. In practical applications the last issue of stability is the primary concern, as the first two conditions are usually postulated.

\subsection{Inferential sensing is an ill-posed problem}

In inferential sensing, none of the conditions for a well-posed problem may be satisfied. First of all, the parameters related to feedwater flow may not provide all of the information which is necessary to estimate a true value of feedwater flow. Second, different parameters can provide different information about the true value of the feedwater flow rate, and finally, feedwater flow estimation can be unstable due to small perturbations in the input data or due to different preprocessing techniques. In the current study we assume that the plant variables selected to infer a true value of the feedwater flow rate do provide information about this value. We also assume that this information is full, and unambiguous, thus postulating existence and uniqueness of the solution for the feedwater inferential sensing problem. Our primary concern in the current study is the stability of the feedwater flow rate estimation, and we shall show that the problem of instability can be solved by regularization.

\subsection{Instability of inferential sensing for feedwater flow evaluation}

To demonstrate the difficulties caused by ill-posed problems, twenty-four variables were selected as predictor variables. These variables are listed in Table 1. These variables were selected based on engineering judgement and on their high correlation with feedwater flow. A linear regression model was then constructed using the predictor variables to estimate the feedwater flow rate ${ }^{1}$. The "training" region for the linear regression model was chosen to be the plant start-up and the first few days of the fuel cycle, when the venturi meter was assumed to be free from corrosion product fouling. Between fuel cycles the venturi meter is removed, acid cleaned, and calibrated; therefore, at the beginning of the fuel cycle, the measured flow rate coincides with the actual flow rate. The fouling during the operation of the NPP increases the pressure drop across the meter. This pressure drop increase results in an overestimation of the feedwater flow rate and thus, overestimation of the reactor's thermal power. The difference between the "true" (estimated) flow rate and the measured flow rate, referred to as drift, can be quantified by using a regression model. In addition, the actual (estimated) flow rate can be used to calculate the actual thermal power of the reactor, allowing the reactor to operate at full licensed power. To evaluate the drift, a check point at a time approximately 6 months into the fuel cycle was chosen.

\footnotetext{
${ }^{1}$ Linear regression was chosen for this demonstration because it is simple to use and illustrates the issues associated with ill-posed problems. The same issues arise in non-linear models, including neural network models.
} 
Table 1 List of 24 variables used as predictor variables to evaluate feedwater flow.

\begin{tabular}{|c|l|c|c|}
\hline Var. Num. & \multicolumn{1}{|c|}{ Description } & Range & Units \\
\hline 1 & FWP Speed & $0-7500$ & RPM \\
\hline 2 & 'A' OTSG EFIC HIGH LEVEL & $0-100$ & PERCENT \\
\hline 3 & FEEDWATER PUMP A SPEED & $0-7500$ & RPM \\
\hline 4 & LINEAR POWER CH NI-6 & $0-125$ & PERCENT \\
\hline 5 & HEATER 3A INLET COND TEMP & $40-300$ & DEGF \\
\hline 6 & HEATER 3B OUTLET COND TEMP. & $40-350$ & DEGF \\
\hline 7 & DEARATOR INLET COND TEMP & $40-350$ & DEGF \\
\hline 8 & HEATER 6A INLET FW TEMP & $40-500$ & DEGF \\
\hline 9 & FWP A DISCHARGE TEMP & $40-500$ & DEGF \\
\hline 10 & FWP A SUCTION TEMP & $40-500$ & DEGF \\
\hline 11 & HEATER 5B OUTLET FW TEMP & $40-500$ & DEGF \\
\hline 12 & STEAM GEN B INLET FW TEMP & $40-600$ & DEGF \\
\hline 13 & HEATER 6B OUTLET FW TEMP & $40-600$ & DEGF \\
\hline 14 & STEAM GEN A LEVEL (OP) & $0-100$ & PERCENT \\
\hline 15 & STEAM GEN A LEVEL (FULL) & $40-640$ & INCHES \\
\hline 16 & STEAM GEN A LEVEL (START UP) & $0-250$ & INCHES \\
\hline 17 & STEAM GEN B INLET FW TEMP & $0-500$ & DEGF \\
\hline 18 & STEAM GEN B LEVEL (START UP) & $0-250$ & INCHES \\
\hline 19 & STEAM GEN A INLET FW TEMP & $40-600$ & DEGF \\
\hline 20 & STEAM GEN B INLET FW TEMP & $40-600$ & DEGF \\
\hline 21 & REHEATER A COLD REHEAT PRESS. & $0-200$ & PSIG \\
\hline 22 & REHEATER D COLD REHEAT PRESS. & $0-200$ & PSIG \\
\hline 23 & REHEATER C COLD REHEAT PRESS. & $0-200$ & PSIG \\
\hline 24 & NO. 2A EXTR LP TURB PRESSURE & $0-20$ & PSIA \\
\hline & & & \\
\hline
\end{tabular}

Prior to any statistical evaluation of the data, a number of preprocessing techniques should be applied to the raw data to ensure consistency of the results. The most common preprocessing techniques are filtering and scaling. It is a well-known fact that least squares models are very sensitive to outliers. To reduce measurement noise we used a median filter with different window sizes. Median filtering has well known outlier rejection and fast digital implementation properties. We are aware of the non-linear nature of median filtering, and precautions have been taken so that correlations between the signals are not changed significantly. Unfortunately, the size of the filter window changes the inferred value of feedwater flow, thus indicating inconsistency of drift evaluations using an ordinary least squares model. The dependence of the drift estimation, at the 6 month check point, on filtering window length is shown in Table 2. Due to different median filter window sizes, the inferred drift can change up to $8 \%$. 
Table 2 Drift dependence on filter window size

\begin{tabular}{|c|c|}
\hline Window length, data points & Drift at check point, klb/hr \\
\hline 0 (no filtering) & 39.50 \\
\hline 3 & 39.50 \\
\hline 5 & 39.46 \\
\hline 7 & 36.13 \\
\hline 11 & 38.72 \\
\hline
\end{tabular}

The second preprocessing technique used was data scaling. It is a well known fact that scaling can change both the ordinary least squares solution, and the regularized solution, for a given problem (Lawson and Hanson, 1974). In the current study we used only column scaling of data matrixes. Each of the 24 predictor variables was scaled based on its maximum anticipated value. These maximum anticipated values are shown in table 1 in the column entitled "Range". For example, the data of variable 1 (feedwater pump speed) were divided by 7500 , which is the maximum anticipated speed of the pump in normal regimes of operation. Prior to scaling, all variables were zero meaned to guarantee an unbiased least squares solution.

After scaling and filtering, the data can be used to build a predictive model to infer feedwater flow rate. In the current study we used the common multivariable linear regression method to develop a predictive model. The 24 variables were regressed on to the response variable (feedwater flow rate) using data from several days at the very beginning of the fuel cycle. Having been estimated, these regression coefficients were used to infer the true value of feedwater flow during the first 7 months of the fuel cycle. The data points were sampled in 30 minute increments. Unfortunately, as it was found out, the value of the drift is very much dependent on the number of data points used to calculate the regression coefficients, again pointing to inconsistency of inference. This dependence is shown in Table 3.

Table 3 Drift dependence on the number of data points used to estimate regression coefficients.

\begin{tabular}{|c|c|}
\hline Number of data points & Drift, klb/hr \\
\hline 200 (approximately 4 days of operation) & 25.08 \\
\hline 300 & 16.25 \\
\hline 400 & 9.82 \\
\hline 500 & 11.98 \\
\hline 600 & 39.46 \\
\hline 700 & 42.86 \\
\hline 800 & 42.02 \\
\hline
\end{tabular}


As can be seen from this table, the drift value depends drastically on the number of "training" points used to estimate the regression coefficients. This is one more source of inconsistency in inferential measurements, which can lead to incorrect inferences about the true value of feedwater flow rate.

The last source of inconsistency in inferential sensing is the data perturbations or noise. Under some conditions the noise can make inferential sensing extremely unstable. The inferential measurement of feedwater flow rate sensor drift is based on the inferred value of the actual feedwater flow rate. The actual flow is inferred based on its relationships to other correlated plants parameters. The problem with using these parameters as predictors is that they are not only highly correlated with feedwater flow rate, but they are also correlated with each other. If this degree of correlation is quite high, the data matrix becomes ill-conditioned and we face the problem which is commonly referred to in statistical literature as "collinearity". Two variables are collinear if the data vectors representing them lie on the same line (i.e., subspace of dimension one). More generally, $\mathrm{k}$ variables are collinear if the vectors that represent them lie in a subspace of dimension less than $\mathrm{k}$; that is, if one of the vectors is a linear combination of the others. In practice, such "exact collinearity" rarely occurs due to noise. A broader notion of collinearity is therefore needed to deal with the problem as it affects statistical estimation. A less strict definition would be that two variables are collinear if they lie almost on the same line, or if the angle between them is small. In the event that one of the variables is not constant, this is equivalent to saying that they have a high degree of correlation between them.

To evaluate the consistency of the inferential drift estimation system under small perturbations in the data, a bootstrap technique was used (Efron, 1982). The bootstrap technique is a statistical method used for evaluating the precision of the regression coefficients or fitted values. If we have $\mathrm{n}$ training data samples, the bootstrap technique randomly selects $\mathrm{n}$ values from both predictor and response variables with replacement, thus providing a bootstrap sample of size $\mathrm{n}$ but with some original values duplicated and some missing. This bootstrap sample is used to regress predictor variables onto the response variable using the same fitting procedure as for the original sample. When the method is repeated a large number of times, the bootstrap procedure provides a set of fitted values whose variability can be estimated and whose sampling distribution can be plotted. This graphical representation demonstrates the precision of the evaluated statistical parameter.

In this study the input data matrix $X$ is of size $600 \times 24$, where 24 is the number of predictor variables and 600 is the number of data samples. The output vector $y$ is of size $600 x 1$, containing 600 corresponding samples of the response variable (feedwater flow rate). The condition number (the ratio of largest to smallest singular values) of the input data matrix is 705 which indicates ill-conditioning, or collinearity of the data. Most well-conditioned matrices have condition numbers well below 100. To check the stability of drift inference, 100 bootstrap 
samples were generated from the original data. For each bootstrap sample, the regression coefficients were estimated using ordinary least squares and test data were used to infer the drift value at a check point approximately 6 months into the fuel cycle. The predicted drift values were used to calculate a probability density function (PDF) of the drift estimates and their standard deviation.

The PDF for the drift estimates is shown in Figure 2. The PDF for the drift value has a large variance, and even worse, it is multimodal. We can see that estimated drift values range from 20 up to nearly $60 \mathrm{klb} / \mathrm{hr}$ with a standard deviation of $6.51 \mathrm{klb} / \mathrm{hr}$. These results indicate the instability of the solution using ordinary least squares, and the resulting inconsistency of the drift estimation. This inconsistency is due to the high sensitivity of the ordinary least squares solution to small perturbations in the data, which is a direct result of the ill-conditioned nature of the problem. To stabilize the drift prediction, a method of regularization should be used to alleviate the ill-conditioning problem.

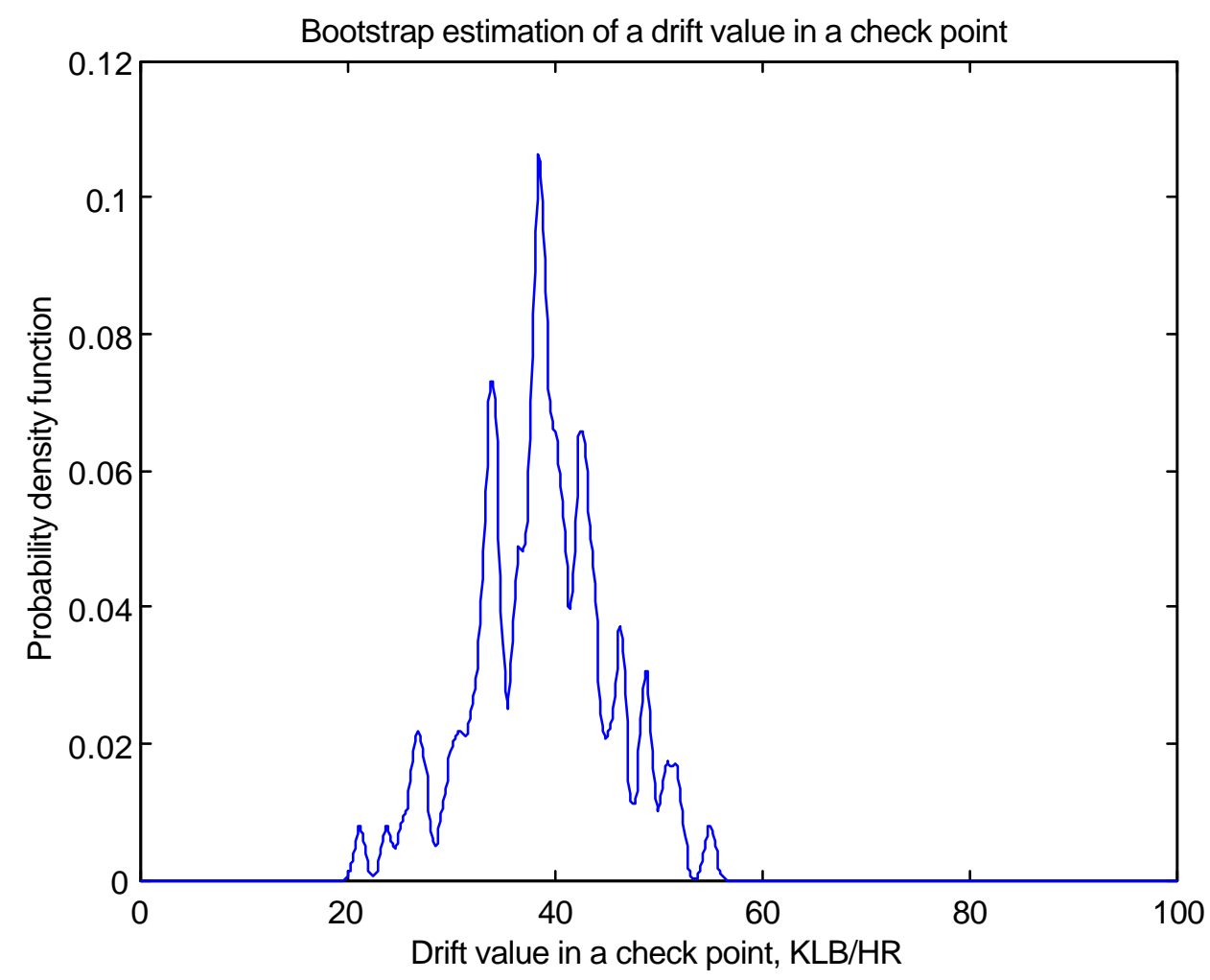

Fig. 2 Instability of drift estimation due to perturbations in the data.

\section{REGULARIZATION}

Prior to considering regularization we have to understand what causes the instability of the ordinary least squares solution. To understand the essence of ill-posed problems for drift detection, let us consider the linear least squares problem. The objective of linear least squares 
is to find a linear combination of predictor variables that provides an accurate estimation of the response variable. Hence, we minimize the square of the difference between $\mathrm{Xw}$ and y, i.e.

$$
\min \|X w-y\|_{2}^{2}, \quad X \in R^{m x n}, m \geq n
$$

With respect to the current application, $\mathrm{X}$ is a data matrix containing predictor variables related to feedwater flow rate, $\mathrm{y}$ is a vector of measured values of feedwater flow rate and $\mathrm{w}$ is a vector of regression coefficients. A very valuable tool in the analysis of ill-posed problems is singular value decomposition (SVD) (Golub and Van Loan, 1996). The SVD of data matrix X can be written as:

$$
\mathrm{X}=\mathrm{U} \Sigma \mathrm{V}^{\mathrm{T}}=\sum_{\mathrm{i}=1}^{\mathrm{n}} \mathrm{u}_{\mathrm{i}} \sigma_{\mathrm{i}} \mathrm{v}_{\mathrm{i}}^{\mathrm{T}}
$$

The components $u_{i}$ and $v_{i}$ are the left and right singular vectors of $X$, and $\sigma_{i}$ are the singular values of the matrix $X$. Assuming that matrix $X$ has a full rank of $n$ in terms of the SVD, the solution for problem (2) can be written as:

$$
\mathrm{w}_{\mathrm{LS}}=\sum_{\mathrm{i}=1}^{\mathrm{n}} \frac{\mathrm{u}_{\mathrm{i}}^{\mathrm{T}} \mathrm{y}}{\sigma_{\mathrm{i}}} \mathrm{v}_{\mathrm{i}}
$$

Equation (4) gives insight into the essence of ill-conditioning. The division by small singular values results in the amplification of high-frequency oscillations of the right singular vectors of the data matrix X. To deal with ill-conditioned problems several methods have been developed which essentially damp or filter out these high frequency oscillations. These methods are called regularization methods because they regularize or smooth potentially unstable least squares solutions. The simplest regularization method is the truncated SVD (TSVD) method. This method truncates the sum in equation 4 at some value $k<$ n, eliminating small singular values from the denominator. The two heuristics used in this method of regularization are as follows:

1. The singular values have a distinct gap in their spectrum. The location of this gap on the singular values curve can be a natural choice for the truncation parameter $\mathrm{k}$.

2. The left and right singular vectors $\mathrm{u}_{\mathrm{i}}$ and $\mathrm{v}_{\mathrm{i}}$ tend to have more sign changes in their elements as the index $i$ increases, i.e., as $\sigma_{i}$ decreases (Hansen, 1997).

Heuristic 2 is only guaranteed to hold for totally positive matrices (Hansen, 1995). A matrix is totally positive if all of its minors of any order are positive (Gantmacher, 1959). Matrices that arise in most practical applications are usually totally positive, but it is important to verify this property prior to the application of any regularization method based on heuristic 2 . TSVD regularization is especially appropriate for ill-conditioned problems which have a large gap (say two orders of magnitude) between two consecutive singular values $\sigma_{\mathrm{i}}$ and $\sigma_{i+1}$. These 
kinds of problems are said to have a well-determined numerical rank. Not all real world problems have a well-determined numerical rank. If the singular value spectrum has no distinct gap, then the problem has an ill-determined numerical rank, and the choice of the truncation or regularization parameter is not as evident as in the former case. But as it was stressed in Hansen (1989), the success of TSVD depends on the satisfaction of the Discrete Picard Condition (DPC) (which assures that the regularized solution exists ), (Hansen, 1990) and not on the existence of a distinct gap in the singular value spectrum of the data matrix X. To deal with ill-conditioned problems having ill-determined numerical rank, the method of regularization proposed by Tikhonov (1963) can be used. In this method, the minimization problem (2) is replaced by the following augmented functional:

$$
\min \left(\|\mathrm{Xw}-\mathrm{y}\|_{2}^{2}+\lambda^{2}\|\mathrm{Lw}\|_{2}^{2}\right)
$$

The regularization parameter $\lambda$, controls the trade-off between the smoothness of the solution and its fit to the data. $\mathrm{L}$ is a well conditioned matrix; for example, a discrete approximation of the derivative operator. The main assumption behind Tikhonov regularization is that the solution should be smooth or non-oscillating. In the case of $\mathrm{L}=\mathrm{I}$, where $\mathrm{I}$ is identity matrix, the Tikhonov's functional (5) is said to be in standard form and is known in statistical literature as "ridge regression" (Hoerl and Kennard, 1970). In this case, we can write the regularized solution as:

$$
w_{\lambda}=\sum_{i=1}^{n} f_{i} \frac{u_{i}^{T} y}{\sigma_{i}} v_{i}
$$

The components $u_{i}$ and $v_{i}$ are the left and right singular vectors of the data matrix $X, \sigma_{i}$ are the singular values of this matrix and $f_{i}=\frac{\sigma_{i}^{2}}{\sigma_{i}^{2}+\lambda^{2}}$ are the filter factors. The role of filter factors is to suppress the contribution of minor components to the solution, thus providing a more stable non-oscillating solution. In Tikhonov regularization the filter factors for large $\sigma_{\mathrm{i}}$ are close to 1 and for small $\sigma_{\mathrm{i}}$ they tend toward zero, thus providing necessary filtering of minor components. In Tikhonov regularization heuristic 2 is the same as was previously stated for the TSVD method, thus ensuring the smoothness or stability of the regularized solution. However, heuristic 1 now states that the singular value spectrum decays to zero without any particular gap in the singular values.

It should be noted that in any practical situation the singular value spectrum does not decay to zero but levels off at some index " $\mathrm{i}$ " due to unavoidable measurement errors or instrumentation noise. The noise level in both the right and left parts of $\mathrm{Xw}=\mathrm{y}$ is a crucial factor for satisfaction of the Discrete Picard Condition and thus for the existence of a "good" regularized solution which is a reasonable approximation to a desired true solution. 
With these theoretical considerations in mind we will now tackle the problem of drift detection in feedwater flow instrumentation. This problem of inferential or virtual measurements is an inverse problem, where the aim is to recover or "infer" about unknown parameters of a physical system from other correlated parameters which are corrupted by measurement noise. In this paper a linear approach to the drift detection problem was adopted because it allows a clear analysis of the regularized solution. Nonlinear methods, such as neural networks, are much more difficult to regularize due to the absence of a consistent, unifying theory of nonlinear regularization.

\subsection{Regularization of feedwater flow evaluation}

A standard form of Tikhonov regularization, known in statistics as ridge regression, was used to regularize the problem. Prior to applying this form of regularization, the regularization parameter $\lambda$ must be chosen to resolve the subtle compromise between the smoothness of the regularized solution and the solution bias. This biasing towards small regression coefficients is the "price" paid for the smoothness of the regularized solution. We want to obtain the smoothest solution possible, without significantly biasing our solution.

Several methods were proposed to determine the optimal regularization parameter. The principle of discrepancy by Morozov (1966), requires the knowledge of the right hand side error e of the equation $\mathrm{Xw}=\mathrm{y}+\mathrm{e}$. When a good estimation of e is available, this method yields a good regularization parameter. Two other highly regarded methods for regularization parameter selection do not assume any knowledge about the error level, but are based on the extraction of information from the data. The generalized cross-validation method, (Golub, Heath and Wahba, 1979) is based on the assumption that if an arbitrary element $y_{i}$ (of the right-hand side y) is removed, then the corresponding regularized solution should predict this observation well (Hansen, 1994). However, the most common method of determining the regularization parameter is the L-curve method (Hansen, 1992). This method proposes that the optimal regularization parameter occurs at the "corner" of a plot of solution norm vs. residual norm. After evaluating the above methods, we found the L-curve to be the most reliable and simple and it was adopted as the method for choosing the regularization parameter in our study. The truncated SVD regularization was also used to assure that different regularization methods provide similar regularized solutions.

The L-curve for the ill-posed problem of drift detection is plotted in Figure 3, for a data matrix containing 600 data points. It should be noted that Figure 3 is a log-log plot because the singular values span three orders of magnitude. An analysis of the curve shows that the best $\lambda$, corresponding to the "corner" of the L-curve, is 0.1275 . It should be pointed out that the proper choice of regularization parameter is a tough problem, and the "optimal" value provided by an L-curve should not be taken blindly. We found that for the drift detection problem the L-curve provided a slightly underregularized solution, so we used a slightly higher value of the regularization parameter than that prescribed by the L-curve. We want to stress that the regularization parameter is a function of the data matrix and should be recalculated each time the training data matrix is changed. The filter factors for $\lambda=0.1275$ are shown in Figure 4 . As can 
be seen from this figure, the contribution of minor components will be heavily damped through filter factor weighting, allowing only the first principal component to remain unfiltered.

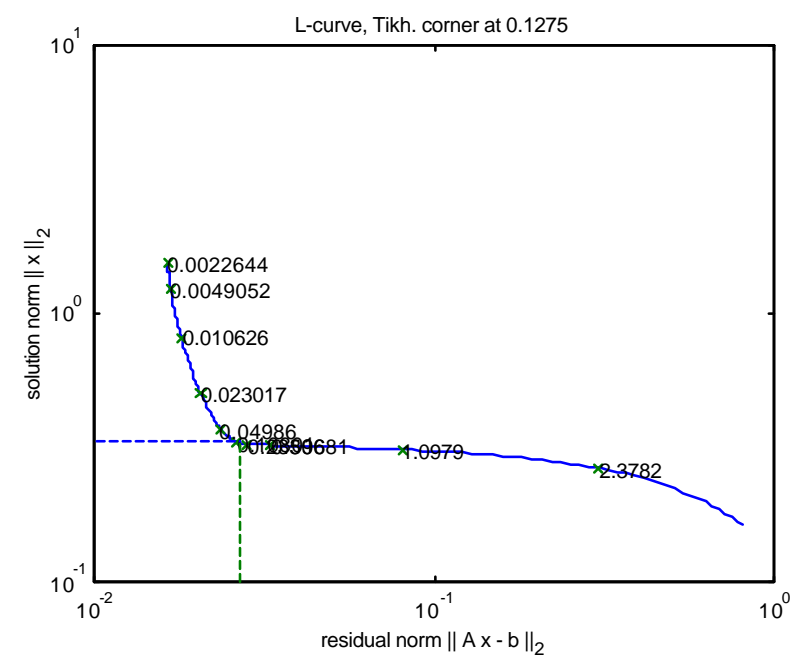

Fig.3 L-curve for Tikhonov regularization

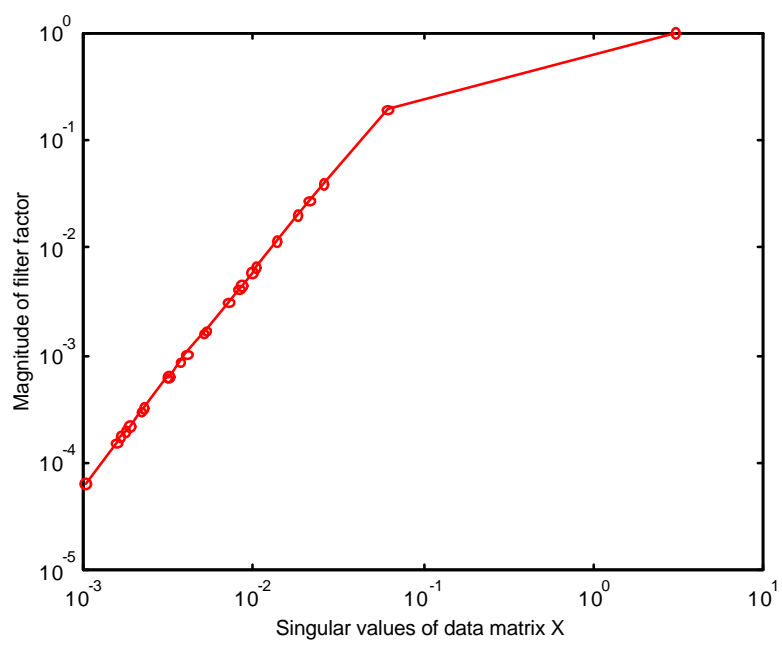

Fig.4 Filter factors as function of the singular values of the data matrix $X$.

Having chosen the optimal regularization parameter we can now calculate the regularized solution, and repeat the tests we performed on the unregularized solution; namely, check the drift dependence on the size of the median filter window, the number of training points and data perturbations. The drift dependence on median filter window size after regularization is shown in Table 4. 
Table 4 Drift dependence on filter window size after regularization

\begin{tabular}{|c|c|}
\hline Window length, data points & Drift at the check point, $\mathrm{klb} / \mathrm{hr}$ \\
\hline 0 (no filtering) & 39.68 \\
\hline 3 & 39.68 \\
\hline 5 & 39.68 \\
\hline 7 & 39.90 \\
\hline 11 & 39.82 \\
\hline
\end{tabular}

This table should be compared with Table 2 where drift dependence on filtering window for the unregularized solution is shown. Comparison of these tables shows that regularization decreased the variability of drift estimation. The changes after regularization are even more dramatic for the drift dependence on the number of training points and on data perturbations. The drift dependence on the number of training points is shown in Table 5.

Table 5 Drift dependence on the number of training points for two regularization methods.

\begin{tabular}{|c|c|c|c|c|}
\hline $\begin{array}{c}\text { Number of data } \\
\text { points }\end{array}$ & $\begin{array}{c}\text { Ordinary least } \\
\text { squares solution } \\
\text { (no } \\
\text { regularization), } \\
\text { drift, klb/hr }\end{array}$ & $\begin{array}{c}\text { Tikhonov } \\
\text { regularization } \\
\text { drift, klb/hr }\end{array}$ & $\begin{array}{c}\text { Regularization } \\
\text { parameter } \lambda \text { for } \\
\text { Tikhonov } \\
\text { regularization }\end{array}$ & $\begin{array}{c}\text { Truncated SVD } \\
\text { regularization, } \\
\text { regularization } \\
\text { parameter k=1, } \\
\text { klb/hr }\end{array}$ \\
\hline 200 & 25.08 & 39.73 & 0.2 & 40.53 \\
\hline 300 & 16.25 & 39.65 & 0.25 & 39.99 \\
\hline 400 & 9.82 & 39.41 & 0.25 & 39.98 \\
\hline 500 & 11.98 & 39.13 & 0.35 & 39.11 \\
\hline 600 & 39.46 & 39.68 & 0.35 & 39.50 \\
\hline 700 & 42.86 & 39.68 & 0.35 & 40.07 \\
\hline 800 & 42.02 & 39.23 & 0.35 & 39.74 \\
\hline 900 & 39.00 & 39.06 & 0.35 & 39.69 \\
\hline
\end{tabular}

For convenience, in the second column we show the results of drift inference without regularization. Analysis of Table 5 reveals that regularization drastically reduced the drift estimation's variability due to using different numbers of training points to calculate the regression coefficients. In fact, regularization made the drift evaluation consistent and invariant under 
changes in the number of training data points. Based on the unregularized solution (column two), it is practically impossible to draw any conclusion about the inferred drift value. On the other hand, the regularized solutions provide highly consistent results. It should be pointed out that both regularization methods give similar results indicating that the true drift value can be inferred based on the current data.

To show how regularization works to stabilize drift measurements under data perturbations we repeat the bootstrap test for the regularized solution. The results of the application of the bootstrap technique using regularization are shown in Figure 5. As can be seen from this figure, the standard deviation of the drift estimation was reduced more than 40 times. The stability of the regularized drift is clearly seen from the unimodal nature of the PDF. This figure should be compared with Figure 2. The mean value of the regularized drift was found to be $39.48 \mathrm{klb} / \mathrm{hr}$. This corresponds to $0.73 \%$ drift in the first six months of operation. This value coincides with previous studies of feedwater flow rate drift estimation and is reasonable from an engineering point of view. The measured and estimated feedwater flow rates are shown in Figure 6. The measured feedwater flow is the upper curve which is maintained stationary to be within regulatory limits. The lower curve is the inferred feedwater flow which is clearly decreasing, indicating fouling of the venturi meter. The true value of feedwater flow rate is represented by this lower curve, which shows that the reactor is losing some power due to derating. Knowledge of this true value would allow the operator to avoid derating and still be within regulatory safety limits.

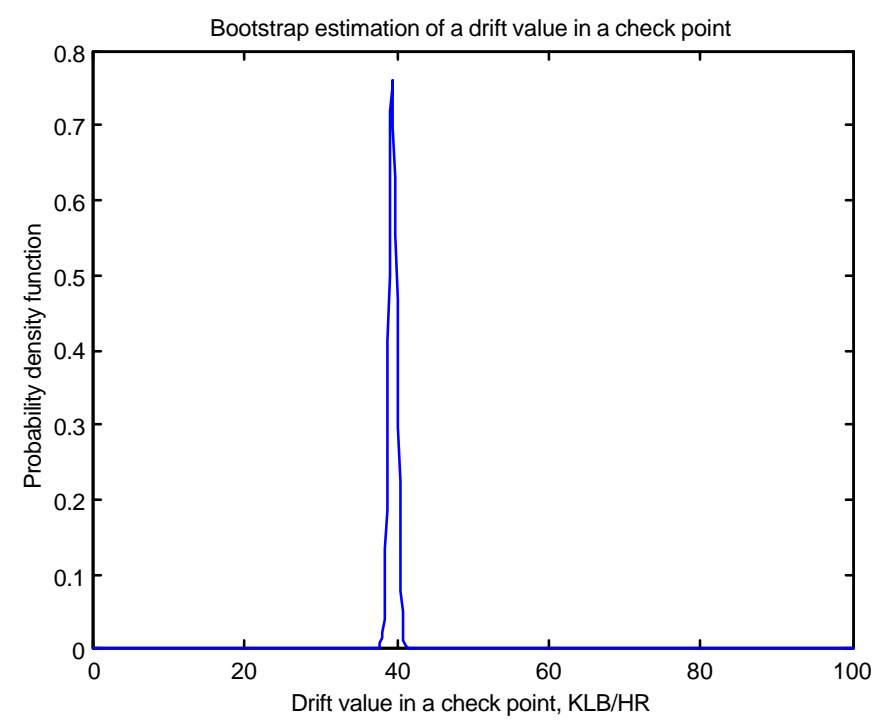

Fig. 5 Regularized estimation of drift value at the check point 


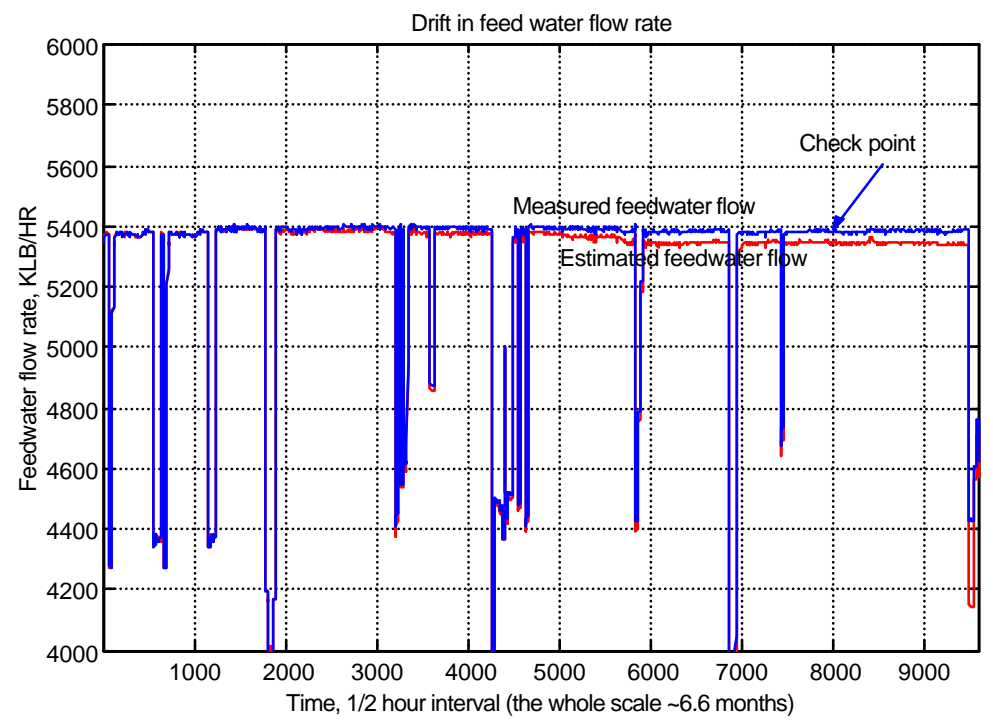

Fig.6 The measured and estimated regularized feedwater flow rates.

\section{CONCLUSIONS}

Inferential sensing is an ill-posed problem which suffers from solution instability when applied to feedwater flow rate estimation. This instability is caused by the ill-conditioning of the data matrix and manifests itself as a non-smooth least squares solution, which is overly sensitive to noise in the data. Regularization is a method which can be used to provide a stable and consistent drift estimation that does not depend on the noise in the data. The regularized solution also remains invariant when different preprocessing techniques are applied, and when different data sets are used to build the predictive model. Thus, the use of regularization in inferential measurements provides an inexpensive, competitive alternative to existing methods for the accurate evaluation of the feedwater flow rate.

\section{NOMENCLATURE}

$\mathrm{Q}_{\mathrm{c}}$ core thermal power

$\mathrm{m}_{\mathrm{fw}}$ feedwater flow rate

$\mathrm{h}_{\mathrm{s}}$ steam enthalpy

$\mathrm{h}_{\mathrm{fw}}$ feedwater enthalpy

$\mathrm{X}$ data matrix of predictor variables

y response variable (feedwater flow)

$\mathrm{W}$ vector of regression coefficients

$\mathrm{U}$ matrix of left eigenvectors of data matrix $\mathrm{X}$

$\mathrm{V}$ matrix of right eigenvectors of data matrix $\mathrm{X}$

$\Sigma$ diagonal matrix of singular values of data matrix $\mathrm{X}$

$\mathrm{u}_{\mathrm{i}}$ left singular vector

$\mathrm{v}_{\mathrm{i}}$ right singular vector 
$\sigma_{\mathrm{i}}$ singular value

$\lambda$ regularization parameter for Tikhonov regularization

$\mathrm{f}_{\mathrm{i}}$ filter factors

e noise

L well-conditioned matrix

PWR pressurized water reactor

BWR boiling water reactor

\section{Subscripts}

c core

S steam

fw feedwater

LS least squares

$\lambda$ - regularized least squares solution using regularization parameter $\lambda$

\section{ACKNOWLEDGEMENTS}

We would like to acknowledge Florida Power Corporation for providing the data, and Idaho National Engineering and Environmental Laboratory for funding this research. We also would like to acknowledge the use of the Matlab freeware package for analysis and solution of discrete ill-posed problems (Hansen, 1994).

\section{REFERENCES}

Chan, A.M.C., Ahluwalia, A.K., Feedwater flow measurement in U.S. nuclear power generation stations, EPRI TR-101388, Electric Power Research Institute, Nov. 1992.

Efron, B., 1982. The Jacknife, The Bootstrap, and Other Resampling Plans, Philadelphia, Penn.: Society for Industrial and Applied Mathematics

Gantmacher, F.R., 1959. Applications of the Theory of Matrices, Interscience Publishers, New York, p.118.

Golub, G.H., and Van Loan, C.F., 1996. Matrix Computations, Third Edition, the Johns Hopkins University Press, Baltimore, MD, pp 70-80.

Golub, G.H., Heath, M.T., and Wahba, G. 1979. , Generalized cross-validation as a method for choosing a good ridge parameter, Technometrics, 21 pp. 215-223.

Hadamard, J., 1923. Lectures on Cauchy's Problem in Linear Partial Differential Equations, Yale University Press, New Haven.

Hansen, P.C., 1997. Rank-Deficient and Discrete Ill-Posed Problems. Numerical Aspects of Linear Inversion, SIAM, Philadelphia, p.20. 
Hansen, P.C., 1995. Test matrices for regularization methods, SIAM J. SCI. Comput. vol. 16, No. 2, March, pp. $506-512$.

Hansen, P.C.,1989. Regularization, GSVD and truncated GSVD, BIT 29 pp. 491-504.

Hansen, P.C., 1990. The discrete Picard condition for discrete ill-posed problems, BIT 30, pp. 658-672.

Hansen, P.C. , 1994. Regularization tools: A Matlab package for analysis and solution of discrete ill-posed problems, Numer. Algorithms, 6 pp. 1-35.

Hansen, P.C. , 1992. Analysis of discrete ill-posed problems by means of the L-curve, SIAM Review vol. 34, No.4, pp. 561-580.

Hoerl, A.E., Kennard, R.W., 1970. Ridge regression: biased estimation for nonorthogonal problems. Technometrics, 12, pp.55-67.

Kavaklioglu, K., and Upadhyaya, Belle R., 1994., Monitoring feedwater flow rate and component thermal performance of pressurized water reactors by means of artificial neural networks. Nuclear Technology Vol. 107, July, pp.112-123

Lawson, C.L., Hanson, R.J., 1974. Solving Least Squares Problems, Prentice - Hall, pp. $183-194$.

Morozov, V.A., 1966. On the solution of functional equations by the method of regularization, Soviet Math. Dokl., 7, pp.414-417

Tikhonov, A.N., 1963. Solution of incorrectly formulated problems and the regularization method, Doklady Akad. Nauk USSR 151, pp. 501-504. 